Review

\title{
Research progress in the role and mechanism of Cadherin-11 in different diseases
}

\author{
Xinyi Chen, Hongjiao Xiang, Shiyu Yu, Yifei Lu, Tao $\mathrm{Wu}^{\varpi}$ \\ Institute of Interdisciplinary Integrative Medicine Research, Shanghai University of Traditional Chinese Medicine, Shanghai 201203, China. \\ $\triangle$ Corresponding author: Tao Wu, PhD, Assistant Professor, Institute of Interdisciplinary Integrative Medicine Research, Shanghai University of Traditional \\ Chinese Medicine, Shanghai, China. E-mail: tw827@shutcm.edu.cn or wutao001827@163.com; Telephone: +86-21-51322748; Fax: +86-21-51322748. \\ (1) The author(s). This is an open access article distributed under the terms of the Creative Commons Attribution License (https://creativecommons.org/licenses/by/4.0/). \\ See http://ivyspring.com/terms for full terms and conditions.
}

Received: 2020.09.01; Accepted: 2020.11.29; Published: 2021.01.01

\begin{abstract}
Cadherin is an important cell-cell adhesion molecule, which mediates intercellular adhesion through calcium dependent affinity interaction. Cadherin-11 (CDH11, OB-cadherin) is a member of cadherin family, and its gene is situated on chromosome 16q22.1. Increasing lines of researches have proved that $\mathrm{CDH} 11$ plays important roles in the occurrence and development of a lot of diseases, such as tumors, arthritis and so on. CDHIl often leads to promoter methylation inactivation, which can induce cancer cell apoptosis, suppress cell motility and invasion, and can inhibit cancer through $W \mathrm{nt} / \beta$-catenin, AKT/Rho A and NF-KB signaling pathways. This review focused on the current knowledge of CDH11, including its function and mechanism in different diseases. In this article, we aimed to have a more comprehensive and in-depth understanding of $\mathrm{CDH} 11$ and to provide new ideas for the treatment of some diseases.
\end{abstract}

Key words: cadherin-11; epithelial-mesenchymal transition; transforming growth factor

\section{Introduction}

Homotypic cadherin interactions mediated the cell-cell adhesion. Cadherins can gather to form homodimers by a zipper-like mechanism, and the intracellular domain is anchored to the actin cytoskeleton by $\alpha$-catenin and $\beta$-catenin. Cadherins are transmembrane $\mathrm{Ca}^{2+}$-dependent homophilic adhesion receptors, which are vital for cellular processes, including the proliferation, differentiation and migration of cyto-skeletal organizations, and for maintenance of structural integrity of tissues and homeostasis [1]. They also play important roles in recognition and sorting of cells during development [2]. Six-cadherin cluster, including Cadherin-1 (CDH1), Cadherin-3 (CDH3), Cadherin-5 (CDH5), Cadherin-8 (CDH8), Cadherin-11 (CDH11) and Cadherin-13 (CDH13), are located on chromo-some 16 (16q22.1-16q24.3). In previous studies, CDH1 and CDH13 were proved as functional tumor inhibitory factors, involved in inhibiting the invasion, proliferation and metastasis of tumor cells [3]. Cadherins are divided into type I and type II, and
CDH11 is a latter type, which is broadly expressed in mesenchymal stem cells (MSCs), smooth muscle cells (SMCs), fibroblasts and osteoblasts. CDH11 expression is very important during epithelial mesenchymal transition (EMT). The proliferation and survival of some cells, including vascular SMCs, osteosarcoma and glioblastoma, are also strongly correlated with the expression of CDH11 [3,4]. More and more studies have showed that $\mathrm{CDH} 11$ plays important roles in the process of tumor, metabolic diseases, arthritis, skin diseases and other diseases, and may be a potential therapeutic target. Therefore, this article reviews the role and mechanism of CDH11 in the development of various diseases in order to further understand it from a macro perspective.

\section{Structure and function of CDH11}

The extracellular domains of $\mathrm{CDH} 11$ have five repeat sequences, called EC1-EC5, and each of them is consisted of about 80-90 amino acid residues. $\mathrm{Ca}^{2+}$ can combine with this area to turn the domains into a 
club-shaped structure, and then initiate the biological effects of cadherin $[5,6]$. The cytoplasmic tail controls the function of CDH11. It can combine with p120 ctn, $\alpha$-catenin, $\beta$-catenin or plakoglobin ( $\gamma$-catenin) to form complexes, mediating cell adhesion and regulating signal transduction. The compound can further interact with a-actinin, vinculin, zonula occludens- 1 (ZO-1) and the actin cytoskeleton (Fig. 1). The phosphorylation of $\beta$-catenin and p120ctn leads to the separation of cadherins [7]. Alimperti et al. [8] found that CDH11 was necessary to induce the differentiation of MSCs into contractile SMCs. CDH11 regulated the expression of transforming growth factor (TGF)- $\beta 1$ and affected the differentiation of SMCs through TGF- $\beta$ receptor II (TGF- $\beta$-RII) pathway. At the same time, $\mathrm{CDH} 11$ could also activate the expression of serum response factor (SRF) and SMCs protein through Rho-associated protein kinase (ROCK) pathway [9]. Cheng et al. [10] found that TGF- $\beta 1$ increased the expression of CDH11 by activating SMAD2/3-Snail signaling pathway, thus promoting the differentiation of human trophoblast cells. In addition, TGF- $\beta 1$ could also activate some non-classical signaling pathways such as mitogenactivated protein kinase (MAPK) and PI3K/Akt. Activation of PI3K/Akt signaling pathway increased Snail protein expression, PI3K/Akt/Snail axis could promote the growth, migration and invasion of tumor cells [11]. CDH11 extracellular binding domain (CDH11-Fc) could induce the phosphorylation of platelet-derived growth factor receptor (PDGFR)- $a$

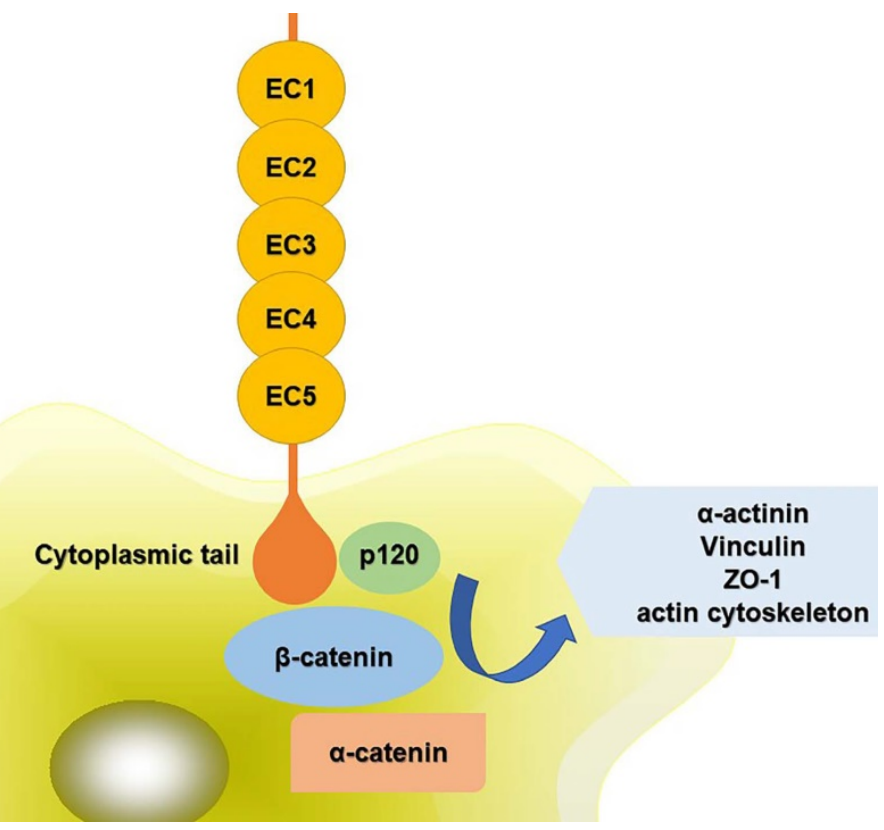

Figure 1. Schematic diagram of $\mathrm{CDH} 1 \mathrm{l}$ mediated cell adhesion. The extracellular domains of $\mathrm{CDH} 11$ have five repeat sequences, called EC1-EC5. The cytoplasmic tail controls the function of CDH11. It can interact with p120ctn, $\alpha$-catenin, $\beta$-catenin to form complexes. And the compound can further interact with $\alpha$-actinin, vinculin, ZO-1 and the actin cytoskeleton. Abbreviations: ZO-1: zonula occludens-1; EC1-5: five extracellular domains of cadherin-11; p120: p120 catenin. fibroblasts and synovial fibroblasts. PDGFR-adependent signal stimulated cell proliferation through phosphorylation of PI3K/Akt and MAPK [12].

The gene of CDH11 is situated in 16q22.1, and its complementary DNA (cDNA) is about $3.8 \mathrm{~kb}$, which contains 16 exons and codes 796 amino acids [5]. Some studies identified CDH11 as the only $1-\mathrm{Mb}$ hemizygous deletion gene detected at 16q22.1. According to the current epigenetic research, CDH11 has often been inactivated by promoter methylation in a variety of tumors. CDH11 inhibits cell invasion and proliferation, induces tumor cell apoptosis, and finally achieves its anti-cancer effect through Wnt/ $\beta$-catenin and AKT/Rho A signaling pathways. Methylation-specific PCR (MSP) analysis has also confirmed the frequent methylation of CDH11 in breast cancer, nasopharyngeal carcinoma, esophageal cancer and other cell lines [3].

\section{The role of CDH11 in different diseases}

It has shown that $\mathrm{CDH} 11$ plays significant roles in different diseases, and the detailed mechanisms including involved signaling pathways are summarized in Table 1.

\section{Rheumatoid Arthritis (RA) and CDH11}

RA is a chronic inflammatory disease, and the main site of inflammation is synovium. In the process of RA, inflammatory synovium tissue experiences severe remodeling and proliferation, fibroblast proliferation, forming an invasive tissue block, called pannus. Pannus tissue can extend to and attach to cartilage, mediate the degradation of cartilage and bone, and then invade these solid tissues [6]. Pannus tissue is consisted of a large number of fibroblast-like-synoviocytes (FLS) and macrophages. FLS is a kind of joint mesenchymal cells, which consists of 1-3 cells, forms a membrane between articular cavity and fibrous joint capsule, provides nutrition and lubrication for avascular articular cartilage, and plays an important role in normal and inflammatory synovium [13-15]. FLS is the main cell type at the junction of pannus and cartilage erosion, maintaining chronic inflammation which leads to joint destruction [16]. 
Table 1. The mechanism of $\mathrm{CDH} 11$ in different diseases

\begin{tabular}{|c|c|c|c|}
\hline Diseases & Mechanism & Signaling pathways & Reference \\
\hline \multirow[t]{3}{*}{$\overline{\mathrm{RA}}$} & angiotensin $\uparrow \mathrm{FLS} \uparrow$ & & {$[21]$} \\
\hline & $\mathrm{CDH} 11-\mathrm{Fc} \uparrow \mathrm{MMPs} \uparrow$ & PI3K/Akt & $\begin{array}{l}{[9,13,14,23,} \\
27]\end{array}$ \\
\hline & IL-6个 TNF- $\alpha \uparrow$ IL-1 $1 \beta \uparrow$ & MAPK or NF-kB & {$[22,25]$} \\
\hline Invasive & 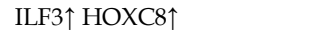 & HOXC8-CDH11-Trio & {$[31,34,35]$} \\
\hline \multirow{2}{*}{\multicolumn{4}{|c|}{$\begin{array}{l}\text { Breast } \\
\text { Cancer }\end{array}$}} \\
\hline & & & \\
\hline TNBC & $\begin{array}{l}\beta \text {-catenin } \uparrow \text { Wnt } 2 \uparrow \text { TCF } 2 \uparrow \\
\text { miR-335 } \downarrow\end{array}$ & Wnt $/ \beta$-catenin & {$[36,37]$} \\
\hline CRC & MC-LR $\uparrow$ & NF-kB & {$[38,39]$} \\
\hline $\mathrm{PCa}$ & VFEEE & $\begin{array}{l}\text { Intracellular signal } \\
\text { pathway }\end{array}$ & {$[43,44]$} \\
\hline $\mathrm{RCC}$ & DNA methylation & & [48] \\
\hline $\begin{array}{l}\text { Pancreatic } \\
\text { cancer }\end{array}$ & EMT $\uparrow$ TGF- $\beta \uparrow$ ECM $\uparrow$ & & {$[3,49-52]$} \\
\hline $\begin{array}{l}\text { cancer } \\
\mathrm{BCA}\end{array}$ & $\begin{array}{l}\text { abnormal hypermethylation } \\
\text { epigenetic inactivation of } \\
\text { CDH11 }\end{array}$ & & {$[53,54]$} \\
\hline GC & $\begin{array}{l}\text { promoter CpG island } \\
\text { hypermethylation }\end{array}$ & & {$[55,56]$} \\
\hline Lung cancer & $\mathrm{ECM} \uparrow$ & & [57] \\
\hline $\begin{array}{l}\text { Head and } \\
\text { neck cancer }\end{array}$ & $\mathrm{ECM} \uparrow$ & & [58] \\
\hline \multirow[t]{2}{*}{ CAVD } & TGF- $\beta 1 \uparrow \alpha-S M A \uparrow$ & ERK1/2-dependent & [62-65] \\
\hline & ECM reconstruction & RhoA/Sox9 & [61] \\
\hline SSc & TGF- $\beta \uparrow$ IFN-I $\uparrow$ ECM $\uparrow$ & Wnt $/ \beta$-catenin & {$[69,70,71]$} \\
\hline Liver fibrosis & TGF- $\beta \uparrow$ ECM $\uparrow$ & & {$[74,75]$} \\
\hline $\begin{array}{l}\text { Pulmonary } \\
\text { fibrosis }\end{array}$ & TGF- $\beta \uparrow$ EMT $\uparrow$ & & {$[71,76,77]$} \\
\hline $\begin{array}{l}\text { Adipose } \\
\text { tissue }\end{array}$ & $\begin{array}{l}\text { CDH11 deficient: } \\
\text { PDGFR }{ }^{\alpha+\uparrow} \text { ILC2s } \uparrow \text { IL-13 }\end{array}$ & & {$[80,81]$} \\
\hline inflammation & M2 macrophages $\uparrow$ & & \\
\hline MI & $\mathrm{IL}-6 \uparrow$ & & [83] \\
\hline \multicolumn{4}{|c|}{$\begin{array}{l}\text { Abbreviations:CDH11:Cadherin-11,OB-cadherin; EMT: epithelial-mesenchymal } \\
\text { transition; RA: Rheumatoid arthritis; FLS: fibroblast-like synoviocytes; CDH11-Fc: } \\
\text { CDH11 extracellular binding domain; MMPs: matrix metalloproteinases; TNF-a: } \\
\text { tumor necrosis factor-a; IL-6: Interleukin-6; IL-13: Interleukin-13; MAPK: } \\
\text { mitogen-activated protein kinase; HOXC8: Homeobox C8; ILF3: interleukin } \\
\text { enhancer-binding factor 3; TNBC: Triple negative breast cancer; TCF: transcription } \\
\text { factor; miR-335: MicroRNA-335; CRC: Colorectal cancer; MC-LR: Microcystin-LR; } \\
\text { NF-kB: nuclear factor-kB; PCa: Prostate cancer; RCC: renal cell carcinoma; TGF: } \\
\text { Transforming growth factor; BCA: bladder cancer; GC: Gastric cancer; CAVD: } \\
\text { Calcified aortic valve disease; a-SMA: a- smooth muscle actin; ECM: extracellular } \\
\text { matrix; SSc: Scleroderma, systemic sclerosis; IFN-I: type I interferon; PDGFR: } \\
\text { platelet-derived growth factor receptor; ILC2s: lymphoid type 2 cells; MI: } \\
\text { Myocardial infarction. }\end{array}$} \\
\hline
\end{tabular}

In joints, CDH11 is mainly expressed in FLS. Studies have shown that $\mathrm{CDH} 11$ can regulate the migration, invasion and degradation of joint tissue, which mediated by FLS, suggesting that CDH11 expressed by FLS plays significant roles in the etiopathogenesis of RA [5,17-19]. Park et al. [20] proved that IL-17 induced the expression of CDH11 in FLS, which could aggravate synovitis and bone destruction. In RA, CDH11 participated in tissue remodeling, led to the aggregation of angiotensin cell clusters, promoted the invasion of angiotensin into articular cartilage, and produced proinflammatory mediators through FLSs in order to enhance the inflammatory response and participate in the inflammatory process of RA [21]. CDH11 and its related intracellular molecular complexes can be used as a molecular tool to allow pannus to extend and invade into articular cartilage pathologically $[13,14]$. FLS not only regulates inflammatory response in synovial microenvironment, but also is a target of inflammatory cytokines [22]. CDH11 can passively mediate cell adhesion, and bind to the same type of cadherin on adjacent cells through the interaction between its extracellular domains. The recombinant soluble form of CDH11-Fc has a high biological activity. It can stimulate synovial fibroblasts in order to produce chemokines, cytokines and matrix metalloproteinases (MMPs), which work together with inflammatory cytokines to promote the activation of synovial fibroblasts, thus promoting the pathogenesis of RA and cartilage erosion [13,14,23], and studies have shown that estrogen can enhance this inflammatory response [24]. The binding of CDH11 on the cell surface to CDH11-Fc can induce the expression of interleukin-6 (IL-6), chemokines and metalloproteinases, which related to the activation of MAPK and nuclear factor- $\mathrm{kB}$ (NF-kB) induced by the Fc tail of immunoglobulin. In another study, CDH11 induced IL-6 production through MAPK signaling or NF- $\mathrm{KB}$ activation, and synergized with other pro-inflammatory molecules such as tumor necrosis factor- $\alpha$ (TNF- $\alpha$ ) and IL-1 $\beta$ to enhance the expression of these inflammatory mediators [22,25]. Yoshioka et al. [26] elucidated the role of CDH11 in TNF-a induced RA-FLS proliferation, which might be a $\beta$-catenin library. Moreover, Wu et al. [27] found that PI3K/Akt pathway was related to the expression of CDH11 induced by pressure or inflammation, which might be involved in the pathogenesis of arthritis [9].

Thus, CDH11 plays a key role in the progression of RA. Proinflammatory cytokines upregulate CDH11, and then promote FLS homophilic interaction mediated by CDH11, leading to inflammation aggravation and diffusion [22]. CDH11 is a potential therapeutic target for RA [28]. However, another study showed that CDH11 targeting regimen was not suitable for RA treatment [29].

\section{Neoplasm and CDH11}

\section{Breast Cancer}

\section{Invasive breast cancer}

In breast cancer, CDH11 can only be expressed in cells and specimens of invasive breast cancer [30], while peripheral $\mathrm{CDH} 11$ expression enhances the migration and metastasis of breast cancer cells, indicating that CDH11 plays a key part in the development of breast cancer [31,32]. The bone metastasis of breast cancer causes significant incidence rate and mortality. In breast cancer cells, up regulation or overexpression of CDH11 may lead to increased bone metastasis in breast cancer [33]. 
A specific transcription factor (TCF) of CDH11, Homeobox $\mathrm{C} 8$ (HOXC8), activates the transcription of CDH11 by directly combining with the TAA-TCC sequence, which located at nucleotide $-196 \sim-191$ of the CDH11 promoter [34]. Recent studies have found the level of HOXC8 in transitional breast cancer cells significantly increases, suggesting that the presence of HOXC8 may affect the migration of breast cancer cells. CDH11 mediates the regulation of HOXC8 on cell migration. The HOXC8-CDH11 axis promotes the membrane localization of Trio, forming a fresh signal axis HOXC8-CDH11-Trio, which can promote Rac activation in metastatic cancer cells, thus promoting the growth, invasion, migration and metastasis of breast cancer cells [35]. Zhang et al. [31] proved that interleukin enhancer-binding factor 3 (ILF3) could combine with CDH11 promoter at nucleotides - 2982 $\sim-2978$ and - 2602 2598, and activate CDH11 transcription by interacting with HOXC8. Further exploring its clinical relevance, it was found that the high expression of HOXC8 and CDH11 was related to the low recurrence free survival rate of breast cancer patients, which further indicated that targeting the HOXC8-CDH11 signal axis was a direction of developing anti metastasis therapy for breast cancer $[34,35]$.

\section{Triple Negative Breast Cancer (TNBC)}

Among all breast cancer cases, the proportion of TNBC is about $10 \%-20 \%$. TNBC has special biological behavior and clinicopathological characteristics, so its prognosis is worse than other types.

Based on the analysis of large data of breast cancer, it was found that the expression of CDH11 was positively correlated with the expression of Wnt signal components such as $\beta$-catenin, Wnt 2 and TCF2, and the expression of CDH11 and $\beta$-catenin in TNBC tissues was high at the same time. $\mathrm{CDH} 11$ regulated the expression level of $\beta$-catenin, and played a key role in enhancing the stem cell, migration and invasion potential of TNBC cells by activating and regulating the typical Wnt-signaling pathway through the $\mathrm{CDH} 11 / \beta$-catenin signal axis [36]. Chen et al. [37] found that there was a negative correlation between CDH11 and MicroRNA-335 (miR-335), exposure to a single specific anti-CDH11 antibody could remarkably increase the ratio of miR-335/ CDH11, inhibit the expression of CDH11, $\beta$-catenin and vimentin, and inhibit the metastasis potential.

By targeting $\beta$-catenin and $\mathrm{CDH} 11$ to regulate the typical Wnt-signaling pathway and inhibit the cancer stem cell (CSC) like phenotype and metastasis phenotype of TNBC cells, it represents a new method of TNBC treatment, which provides a basis for further exploring $\mathrm{CDH} 11$ as a candidate target of TNBC targeted treatment [36,37].

\section{Colorectal cancer (CRC)}

$\mathrm{CRC}$ is one of the most common cancers [38]. Its specific pathogenesis includes the activation of oncogenes and the inactivation of tumor suppressor genes.

Microcystin-LR (MC-LR) is a kind of tumor initiator, which has been confirmed to participate in the occurrence of human primary cancer. The results of PCR displayed that the expression of CDH11 gene increased significantly after MC-LR exposure, while the cell migration and invasion induced by CDH11 gene knockout decreased, suggesting that MC-LR upregulated the expression of CDH11 in HT-29 cells, and enhanced the cell migration and invasion. Therefore, CDH11 is a tumor promoter, which participates in the cytoskeleton reorganization and cell movement induced by MC-LR, so as to enhance the motility and invasiveness of cancer cells. It is suggested that the inhibition of $\mathrm{CDH} 11$ expression may be a new and promising therapeutic strategy for CRC [39].

The inactivation of tumor suppressor genes is closely related to epigenetic changes. DNA of tumor cell lines copy number distortion and $1-\mathrm{Mb}$ hemizygote deletion at 16q21-22.1, and CDH11 is the sole known gene situated in the deletion, so CDH11 may be a candidate tumor suppressor gene related to 16q21-22.1 deletion [3]. In CRC, the down-regulation of CDH11 is caused by promoter methylation, and the level of CDH11 methylation is particularly high in CRC tissues. CDH11 can induce cell periodic arrest and apoptosis in G0 or G1 phase, inhibit the proliferation of CRC cells and formation of colonies, and inhibit tumor cell growth, migration, invasion and proliferation by inactivating NF- $\mathrm{kB}$ signal pathway. Therefore, CDH11 is considered as a functional tumor suppressor gene of CRC, which can be used as a prognostic biomarker of value for further study [38].

\section{Prostate cancer (PCa)}

PCa has been one of common cancers in humans in recent years. Since the proliferation of PCa cells is stimulated by androgen receptor (AR), the main method to treat the advanced PCa now is androgen ablation. However, some patients treated with androgen deprivation therapy eventually became resistant to it and developed bone metastases [40]. Bone metastasis is the leading cause of PCa-related death [41]. It was found that CDH11 was highly expressed in the PC-3 cell line in bone metastases. In addition, when the CDH11 gene was specifically knocked out, the incidence of PC-3 bone metastasis 
was significantly reduced, providing evidence for the correlation between CDH11 and PCa [42]. Satcher et al. [43] confirmed that clathrin mediated the endocytosis of CDH11 through a unique structural motif, the "clathrin-binding motif" VFEEE, thus regulating the migration of $\mathrm{PCa}$ cells mediated by CDH11. Another study further demonstrated that CDH11 mediated the adhesion of PCa cells to osteoblasts, enabling $\mathrm{PCa}$ cells to insert into osteoblasts and promoting the proliferation, invasion and migration of PCa cells. Furthermore, according to microarray analysis, since CDH11-mediated cell invasion and migration were rely on its cytoplasmic domain, it suggested that CDH11 might activate a signaling pathway that promoted the migration of PCa cells to bone [44].

Since bone metastasis often occurs after the progression of castration resistance, studies have shown that androgen depletion can lead to the up-regulation of CDH11, thereby increasing the metastasis of PCa to bone. In other words, treatment strategies that blocked the expression or function of CDH11 may reduce the possibility of bone metastasis in patients undergoing androgen ablation, which provides an idea for clinical treatment [40]. For example, the dimer of CDH11 extracellular domain was expressed as Fc fusion protein (OB-CAD-FC), which can competitively inhibit the combination of osteoblasts and PCa cells [45]. Some studies have focused on the monoclonal antibodies against the extracellular domain of CDH11, targeting two kinds of antibodies, $\mathrm{mAb} 2 \mathrm{C} 7$ and 1A5, which have been proved to be able to significantly reduce bone metastasis in vivo, but the specific mechanism still needs further study [46].

\section{Other cancers}

In addition to these cancers, there are many other cancers that are associated with CDH11.

Consistent with the possibility that CDH11 may participate in bone metastasis in breast cancer and PCa in former researches, studies have indicated that $\mathrm{CDH} 11$ is involved in bone organ-specific metastasis of 786-O cell lines by increasing the migration of renal cell carcinoma (RCC) cells or the adhesion of RCC to osteoblasts in bone marrow [47]. Carmona et al. [48] proved that $\mathrm{CDH} 11$ also played an important role in the early diagnosis of RCC, and CDH11 gene might be a cancer suppressor gene during the initiation and development of RCC. CDH11 gene methylation was associated with tumor invasion and metastasis, suggesting that deletion of CDH11 gene expression caused by DNA methylation was involved in the occurrence and development of a variety of tumors. Therefore, CDH11 gene expression can be induced by demethylation drugs to treat tumors.

CDH11 is also involved in the activation of pancreatic stellate cells (PSCs) and migration of pancreatic cancer cells. CDH11 plays an inflammation-inducing role in EMT and is involved in progression from chronic pancreatitis to pancreatic cancer $[3,49]$. The study detected that CDH11 might be involved in the feedforward mechanism mediating TGF- $\beta$ generation and signal transduction. The expression level of CDH11 was up-regulated in diseased pancreatic tissues, and maintained PSCs activated phenotype $[50,51]$. The synthesis of TGF- $\beta$ and the continuous activation of PSCs led to the over synthesis of extracellular matrix (ECM) and promoted pancreatic fibrosis and metastasis of pancreatic cancer cells [52].

In addition, Lin et al. [53] found that hypermethylation of CDH11 abnormal promoter in bladder cancer (BCA) cells led to the epigenetic inactivation of CDH11, and BCA with high CDH11 expression had poor prognosis [54]. Zhang et al. [55] found that the bidirectional regulation of C12orf59 and CDH11 formed positive feedback, promoted the growth of Gastric cancer (GC) cells, and maintained the metastasis and invasion of human GC cells. Eyvazi et al. [56] also proved promoter $\mathrm{CpG}$ island hypermethylation of CDH11 gene in patients with GC. Studies have shown that the migration of lung cancer cells and head and neck cancer cells can be inhibited by regulating the adhesion of ECM and the expression of CDH11 [57,58]. In addition, CDH11 is one of the potential biomarkers of diffuse large B-cell lymphoma (DLBCL) and plays an important role in predicting central nervous system recurrence [59]. CDH11 is also expressed in stromal cells of ovarian tumors, such as myofibroblasts, vascular SMCs, and endothelial cells [17].

\section{Calcific Aortic Valve Disease (CAVD) and CDH11}

CAVD can cause aortic thickening and hardening, which impairs the fine regulation function and eventually leads to regurgitation and stenosis. The risk of heart disease, atherosclerosis, heart failure and stroke will be increased in patients with early-onset CAVD [60]. Because there are no effective biomarkers to diagnose or delay the disease progression, it is very important to identify the signal pathways that mediate the homeostasis and initiate the disease progression in the valve for the development of potential diagnostic and therapeutic targets [61].

Myofibroblasts play important parts in the development process of CAVD. TGF- $\beta 1$ can induce myofibroblast differentiation of aortic valve 
interstitial cells (AVICs), resulting in the up regulation of a-smooth muscle actin (a-SMA) and CDH11 in an ERK1/2-dependent manner, as well as an increase in contractility, cell-cell connectivity and intercellular tension. Finally, the tension of the whole monolayer cells increases, resulting in the imbalance of production force, which leads to the apoptosis of weak cells and the formation of aggregates as the initial step of morphogenesis of calcified nodules. When the calcified nodules mature, the cells around the aggregates underwent strain amplification due to the non-deformation of the nodules, leading to apoptosis and calcification [62-65].

The mechanism of CDH11 participating in valve calcification and ECM reconstruction in adults is accomplished by RhoA/Sox9 in vivo and in vitro. RhoA and Sox9 are downstream targets of CDH11. Among them, CDH11 increases the sensitivity of cells to mechanical tension through RhoA signal and promotes the differentiation of stromal cells into myofibroblasts and osteoblasts. Up regulation of RhoA and Sox9 leads to excessive mechanical activation and ECM remodeling, leading to morphogenesis that severely impairs valve function. Sox9 can be used as a therapeutic marker to reduce the expression of osteogenic molecules [61]. In addition, studies have found that the lack or knockout of CDH11 can destroy the polarity of valve cells, the formation, migration and matrix compaction of stress fibers, reduce RhoA activity mediated filamentous protrusion [66], prevent valve stenosis, lobular thickening and hardening, and thus prevent CAVD. Clark et al. [67] found that CDH11 caused aortic valve stenosis in Notch1 mutant mice, and blocking CDH11 could prevent valve stenosis, lobular thickening and hardening, and the expression of inflammatory genes. Therefore, targeting CDH11 is a new therapeutic strategy to prevent disease progression in patients with hereditary and idiopathic CAVD.

\section{Skin diseases and CDH11}

Scleroderma (systemic sclerosis, SSc) is a kind of connective tissue disease with multiple system damage, with progressive fibrosis of viscera and skin. The complex etiopathogenesis of SSc involves inflammation and autoimmunity, vascular disease, and excessive deposition of ECM [68]. Dendritic cells, macrophages and $\mathrm{T}$ cells are involved in the activation of fibroblasts and myofibroblasts. Stimulated by TGF- $\beta$, they produce a large number of ECM and lead to tissue fibrosis. SSc involves a variety of pathways, such as Type I interferon (IFN-I), TGF- $\beta$, Wnt- $\beta$ catenin and CDH11 [69].

CDH11 can induce macrophages to produce TGF- $\beta$ and regulate the level of TGF- $\beta$ in vivo, thus indirectly regulating the behavior of fibroblasts. It is a complex process during the progression of tissue fibrosis in SSc patients, which involves many molecules in a variety of cell types. Early endothelial dysfunction, as well as the activation and recruitment of inflammatory cells (including macrophages and $\mathrm{CD} 4{ }^{+} \mathrm{T}$ cells) to the skin can lead to the increase of inflammatory cytokines (including CCL2, IL-6 and IFN-I) and pro fibrogenic cytokines (such as interleukin-13 (IL-13) and TGF- $\beta$ ), thus activating tissue injury and wound healing pathways. Under the action of TGF- $\beta$ and other fibrogenic mediators, fibroblasts aggregate in the fibrotic tissue, resulting in mounting deposition of ECM and tissue fibrosis $[70,71]$.

In conclusion, it has been confirmed that CDH11 is a mediator of skin fibrosis, and anti CDH11 antibody can prevent the development of early inflammatory stage of skin diseases. In addition, CDH11 antagonist can accelerate the resolution of existing fibrosis, suggesting that $\mathrm{CDH} 11$ may be a potential therapeutic target for SSc [70].

\section{Fibrotic diseases and CDH11}

There are no reliable diagnostic biomarkers and effective therapeutic drugs for liver fibrosis, however, more and more studies have been focused on the effect of CDH11 in the pathogenesis of liver fibrosis [72-74]. Liver fibrosis is accompanied with massive deposition of ECM proteins, which are mainly from activated hepatic stellate cells (HSCs). Numerous studies have shown that $\mathrm{CDH} 11$ is expressed on a variety of cells in liver fibrosis, including macrophages, injured hepatocytes and HSCs. CDH11 regulates TGF- $\beta$ production in macrophages, induces HSCs activation, transdifferentiates into highly proliferative myofibroblasts, and secretes a variety of ECM proteins. Excessive ECM destroys normal liver structure, leading to cirrhosis $[74,75]$.

In addition, $\mathrm{CDH} 11$ can also mediate the contact with fibroblasts, and promote pulmonary fibrosis by regulating the production of TGF- $\beta$ in alveolar macrophages and EMT in alveolar epithelial cells $[71,76,77]$. CDH11 even participates in the development of intestinal fibrosis and renal fibrosis [78], suggesting that CDH11 is a common mediator of fibrosis in various tissues, as well as indicating that targeting CDH11 used in the treatment of fibrosis diseases is worthy of further study [75].

\section{Other diseases and CDH11}

Adipose tissue inflammation is a metabolic disease that drives the development of insulin resistance and diabetes in obese patients [79]. Many cells contribute to the control of adipose tissue 
inflammation, such as M2 macrophages, eosinophils, Tregs, innate lymphoid type 2 cells (ILC2s), and invariant NKT (iNKT) cells. The study found that the production of PDGFR $^{\alpha+}$ fibroblasts of interleukin-33 (IL-33) was increased in CDH11 deficient mice, while the proliferation of M2 macrophages, ILC2s and IL-13 was enhanced. IL-33 induced the activation and proliferation of ILC2s to express IL-13 and interleukin-5 (IL-5), which induced M2 macrophage and eosinophil aggregation, as well as regulated Treg homeostasis of adipose tissue, contributing to the control of adipose tissue inflammation. Experiments have also demonstrated that CDH11 deficiency significantly prevents adipose tissue inflammation and metabolic syndrome in obese mice [80,81]. Therefore, CDH11 targeting is of great significance in the treatment of adipose tissue inflammation, diabetes and metabolic syndrome.

Myocardial infarction (MI) can remarkably reduce cardiac function and may exacerbate the development of heart failure. The process of infarct healing begins with the involvement of resident cells and newly recruited cells in healing, remodeling, and cardiac stabilization, followed by coordinated clearance and replacement of injured tissue by immune cells to form a dense, robust and stable collagen scar that maintains the integrity of the myocardial wall to prevent cardiac rupture [82]. Studies have shown that cells expressed by CDH11 lead to inflammation-driven fibrotic remodeling after MI through regulating the recruitment of bone marrow-derived cells, then limiting fibroblast-induced IL-6 expression and promoting vascularization, thereby improving prognosis. It suggests that $\mathrm{CDH} 11$ plays an important part in solving tissue rupture and promoting myocardial remodeling. CDH11 may serve as a potential therapeutic target, so targeting $\mathrm{CDH} 11$ may reduce early inflammation and tissue breakdown mediated by neutrophil and macrophage, later collagen deposition mediated by myofibroblast and inflammation and fibrotic remodeling after MI [83].

\section{Conclusion and Perspectives}

At present, DNA demethylation is an important way to restore tumor suppressor gene function [84]. CDH11 can inactivate promoter methylation. Therefore, increasing researchers have carried out related research on $\mathrm{CDH} 11$ targeted disease treatment. They explored the targeted therapy of CDH11 by controlling gene knockout and screening anti-CDH11 antibody. In cancers, previous studies have shown that knockout of CDH11 gene can reduce the size of the formed mammary bulb and inhibit breast cancer tumorigenesis [36]. Chen et al. [37] treated human metastatic breast cancer cell lines MCF7 and MDA-MB-231 with a single specific anti-CDH11 antibody mediated by miR-335, which proved that the antibody reduced the $\mathrm{CD} 44^{\text {hi }} \mathrm{CD} 24^{\mathrm{neg} / \mathrm{lo}}$ cell population, inhibited the motility of breast cancer cells and the efficiency of mammary bulb formation. Lee et al. [46] studied two kinds of antibodies, mAb 2C7 and 1A5, which blocked CDH11 mediated cell adhesion, and determined that the 343-348 motif of EC3 could be used as a good target for the treatment of CDH11 antibody. It was proved that they can significantly reduce bone metastasis in vivo and achieve the treatment of PCa. In CAVD, Clark et al. [67] found that targeting CDH11 could prevent the disease progression of hereditary CAVD caused by Notch1 mutation. In skin diseases, some studies have shown that anti-CDH11 monoclonal antibody can inhibit $\mathrm{CDH} 11$, and regulate TGF- $\beta$ level in vivo and TGF- $\beta$ production by macrophages, which can effectively reduce skin fibrosis and accelerate the resolution of existing fibrosis [70,71]. In addition, there are many studies on the role of blocking CDH11 in RA and metabolic diseases.

CDH11, as a member of the cadherin superfamily, is a group of transmembrane proteins that are mainly located at the junction of adhesion molecules and mediate homophilic intercellular adhesion [51]. It can regulate multiple aspects of cell behavior, including proliferation, differentiation, apoptosis, cell polarity, self-renewal and differentiation of embryonic stem cells, as well as maintenance of tissue integrity [1], which plays important roles in multiple cellular tissues. This review focuses on the progress of research on the role and mechanism of CDH11 in the development of various diseases, and discovers some rules of the role of CDH11. In conclusion, CDH11 may be an effective target for the treatment and prevention of various diseases, such as arthritis, cancer, aortic calcification disease, dermatosis, etc. Therefore, CDH11 targeted therapy will become a promising therapeutic strategy. Nowadays, there have been a lot of research results on CDH11 as a target therapy for various diseases, but the specific mechanism of CDH11 targeted therapy needs to be further studied and clarified. More research work is still needed to understand the mechanism of CDH11 acting on these diseases and reveal the molecular pathways guiding these processes, which is of great significance for discovering new therapeutic ideas and methods.

\section{Abbreviations}

CDH11: Cadherin-11, OB-cadherin; CDH1: Cadherin-1, E-cadherin; $\mathrm{CDH} 3$ : Cadherin-3, 
P-cadherin; CDH5: Cadherin-5, VE-cadherin; CDH8: Cadherin-8; CDH13: Cadherin-13, H-cadherin; SMCs: smooth muscle cells; EMT: epithelial-mesenchymal transition; MSCs: mesenchymal stem cells; ZO-1: zonula occludens-1; SRF: serum response factor; ROCK: Rho-associated protein kinase; MAPK: mitogen-activated protein kinase; cDNA: complementary DNA; MSP: methylation-specific PCR; RA: Rheumatoid arthritis; FLS: fibroblast-like synoviocytes; CDH11-Fc: CDH11 extracellular binding domain; MMPs: matrix metalloproteinases; TNF-a: tumor necrosis factor-a; IL-6: Interleukin-6; IL-13: Interleukin-13; IL-33: Interleukin-33; IL-5: Interleukin-5; HOXC8: Homeobox C8; ILF3: interleukin enhancer-binding factor 3; TNBC: Triple negative breast cancer; TCF: transcription factor; miR-335: MicroRNA-335; CSC: cancer stem cell; CRC: Colorectal cancer; MC-LR: Microcystin-LR; PCR: polymerase chain reaction; NF-kB: nuclear factor-kB; PCa: Prostate cancer; AR: androgen receptor; OB-CAD-FC: Fc fusion protein; RCC: renal cell carcinoma; PSCs: pancreatic stellate cells; TGF: Transforming growth factor; BCA: bladder cancer; GC: Gastric cancer; DLBCL: diffuse large B-cell lymphoma; CAVD: Calcified aortic valve disease; AVICs: aortic valve interstitial cells; a-SMA: asmooth muscle actin; ECM: extracellular matrix; SSc: Scleroderma, systemic sclerosis; IFN-I: type I interferon; HSCs: hepatic stellate cells; PDGFR: platelet-derived growth factor receptor; ILC2s: lymphoid type 2 cells; iNKT: invariant NKT cells; MI: Myocardial infarction.

\section{Acknowledgements}

This work was supported by the National Natural Science Foundation of China (81873076), the Shanghai Talents Development Fund Project in China (2017090) and the Innovation Project for Undergraduates of Shanghai University of Traditional Chinese Medicine (2020SHUTCM149).

\section{Competing Interests}

The authors have declared that no competing interest exists.

\section{References}

1. Alimperti S, Andreadis ST. CDH2 and CDH11 act as regulators of stem cell fate decisions. Stem Cell Res. 2015;14(3):270-82.

2. Kawaguchi J, Takeshita S, Kashima T, Imai T, Machinami R, Kudo A. Expression and function of the splice variant of the human cadherin- 11 gene in subordination to intact cadherin-11. Journal of bone and mineral research : the official journal of the American Society for Bone and Mineral Research. 1999;14(5):764-75.

3. Li L, Ying J, Li H, Zhang Y, Shu X, Fan Y, et al. The human cadherin 11 is a pro-apoptotic tumor suppressor modulating cell stemness through Wnt/beta-catenin signaling and silenced in common carcinomas. Oncogene. 2012;31(34):3901-12.
4. Liu Y, Lei P, Row S, Andreadis ST. Cadherin-11 binds to PDGFRbeta and enhances cell proliferation and tissue regeneration via the PDGFR-AKT signaling axis. FASEB J. 2020;34(3):3792-804.

5. Dou C, Yan Y, Dong S. Role of cadherin-11 in synovial joint formation and rheumatoid arthritis pathology. Mod Rheumatol. 2013;23(6):1037-44.

6. Chang SK, Gu Z, Brenner MB. Fibroblast-like synoviocytes in inflammatory arthritis pathology: the emerging role of cadherin- 11 . Immunol Rev. 2010;233(1):256-66.

7. Kashima T, Nakamura K, Kawaguchi J, Takanashi M, Ishida T, Aburatani $\mathrm{H}$, et al. Overexpression of cadherins suppresses pulmonary metastasis of osteosarcoma in vivo. Int J Cancer. 2003;104(2):147-54.

8. Alimperti S, You H, George T, Agarwal SK, Andreadis ST. Cadherin-11 regulates both mesenchymal stem cell differentiation into smooth muscle cells and the development of contractile function in vivo. J Cell Sci. 2014;127(Pt 12):2627-38.

9. Park SJ, Kim KJ, Kim WU, Cho CS. Interaction of mesenchymal stem cells with fibroblast-like synoviocytes via cadherin- 11 promotes angiogenesis by enhanced secretion of placental growth factor. J Immunol. 2014;192(7):3003-10.

10. Cheng JC, Yi Y, Chang HM, Leung PCK. TGF- $\beta 1$ up-regulates cadherin-11 expression through Snail: A potential mechanism for human trophoblast cell differentiation. Cell Signal. 2018;43:55-61.

11. Wang Z, Li J, Long X, Jiao L, Zhou M, Wu K. MRPS16 facilitates tumor progression via the PI3K/AKT/Snail signaling axis. J Cancer. 2020;11(8): 2032-43.

12. Madarampalli B, Watts GFM, Panipinto PM, Nguygen HN, Brenner MB, Noss EH. Interactions between cadherin- 11 and platelet-derived growth factor receptor-alpha signaling link cell adhesion and proliferation. Biochim Biophys Acta Mol Basis Dis. 2019;1865(6):1516-24.

13. Noss EH, Watts GF, Zocco D, Keller TL, Whitman M, Blobel CP, et al. Evidence for cadherin-11 cleavage in the synovium and partial characterization of its mechanism. Arthritis Res Ther. 2015;17(1):126.

14. Kiener HP, Niederreiter B, Lee DM, Jimenez-Boj E, Smolen JS, Brenner MB. Cadherin 11 promotes invasive behavior of fibroblast-like synoviocytes. Arthritis Rheum. 2009;60(5):1305-10.

15. Chang SK, Noss EH, Chen M, Gu Z, Townsend K, Grenha R, et al. Cadherin-11 regulates fibroblast inflammation. Proc Natl Acad Sci U S A. 2011;108(20):8402-7.

16. Mizoguchi F, Slowikowski K, Wei K, Marshall JL, Rao DA, Chang SK, et al. Functionally distinct disease-associated fibroblast subsets in rheumatoid arthritis. Nat Commun. 2018:9(1):789.

17. C VONB, Oliveira-Ferrer L, Löning $\mathrm{T}$, Trillsch F, Mahner $\mathrm{S}$, Milde-Langosch K. Cadherin-11 mRNA and protein expression in ovarian tumors of different malignancy: No evidence of oncogenic or tumor-suppressive function. Mol Clin Oncol. 2015;3(5):1067-72.

18. Kou XX, Li CS, He DO, Wang XD, Hao T, Meng Z, et al. Estradiol promotes M1-like macrophage activation through cadherin-11 to aggravate temporomandibular joint inflammation in rats. J Immunol. 2015;194(6):2810-8

19. Zhao C, Zhang L, Kong W, Liang J, Xu X, Wu H, et al. Umbilical Cord-Derived Mesenchymal Stem Cells Inhibit Cadherin-11 Expression by Fibroblast-Like Synoviocytes in Rheumatoid Arthritis. J Immunol Res. 2015;2015:137695.

20. Park YE, Woo YJ, Park SH, Moon YM, Oh HJ, Kim JI, et al. IL-17 increases cadherin-11 expression in a model of autoimmune experimental arthritis and in rheumatoid arthritis. Immunol Lett. 2011;140(1-2):97-103.

21. Kiener HP, Karonitsch T. The synovium as a privileged site in rheumatoid arthritis: cadherin-11 as a dominant player in synovial pathology. Best Pract Res Clin Rheumatol. 2011;25(6):767-77.

22. Sfikakis PP, Vlachogiannis NI, Christopoulos PF. Cadherin-11 as a therapeutic target in chronic, inflammatory rheumatic diseases. Clin Immunol. 2017;176:107-13.

23. Noss EH, Chang SK, Watts GF, Brenner MB. Modulation of matrix metalloproteinase production by rheumatoid arthritis synovial fibroblasts after cadherin 11 engagement. Arthritis Rheum. 2011;63(12):3768-78.

24. Kou XX, Wang XD, Li CS, Bi RY, Meng Z, Li B, et al. Estradiol-potentiated cadherin-11 in synovial membrane involves in temporomandibular joint inflammation in rats. J Steroid Biochem Mol Biol. 2014;143:444-50.

25. Ding X, Zhang Y, Huang Y, Liu S, Lu H, Sun T. Cadherin-11 involves in synovitis and increases the migratory and invasive capacity of fibroblast-like synoviocytes of osteoarthritis. Int Immunopharmacol. 2015;26(1):153-61.

26. Yoshioka R, Kita Y, Nagahira A, Manno A, Makita N, Tomita U, et al. Quantitative analysis of cadherin-11 and $\beta$-catenin signalling during proliferation of rheumatoid arthritis-derived synovial fibroblast cells. J Pharm Pharmacol. 2015;67(8):1075-82 
27. Wu M, Xu T, Zhou Y, Lu H, Gu Z. Pressure and inflammatory stimulation induced increase of cadherin-11 is mediated by PI3K/Akt pathway in synovial fibroblasts from temporomandibular joint. Osteoarthritis Cartilage. 2013;21(10):1605-12.

28. Sfikakis PP, Christopoulos PF, Vaiopoulos AG, Fragiadaki K, Katsiari C, Kapsimali V, et al. Cadherin-11 mRNA transcripts are frequently found in rheumatoid arthritis peripheral blood and correlate with established polyarthritis. Clin Immunol. 2014;155(1):33-41.

29. Senolt L. Emerging therapies in rheumatoid arthritis: focus on monoclonal antibodies. F1000Res. 2019;8.

30. Pohlodek K, Tan YY, Singer CF, Gschwantler-Kaulich D. Cadherin-11 expression is upregulated in invasive human breast cancer. Oncol Lett. 2016;12(6):4393-8

31. Zhang Y, Yang C, Zhang M, Liu H, Gong C, Zhang J, et al. Interleukin enhancer-binding factor 3 and HOXC8 co-activate cadherin 11 transcription to promote breast cancer cells proliferation and migration. Oncotarget. 2017;8(64):107477-91.

32. Assefnia S, Dakshanamurthy $S$, Guidry Auvil JM, Hampel C, Anastasiadis PZ, Kallakury B, et al. Cadherin-11 in poor prognosis malignancies and rheumatoid arthritis: common target, common therapies. Oncotarget. 2014;5(6):1458-74.

33. Awolaran O, Brooks SA, Lavender V. Breast cancer osteomimicry and its role in bone specific metastasis; an integrative, systematic review of preclinical evidence. Breast. 2016;30:156-71.

34. Li Y, Chao F, Huang B, Liu D, Kim J, Huang S. HOXC8 promotes breast tumorigenesis by transcriptionally facilitating cadherin-11 expression. Oncotarget. 2014;5(9):2596-607.

35. Li Y, Guo Z, Chen H, Dong Z, Pan ZK, Ding H, et al. HOXC8-Dependent Cadherin 11 Expression Facilitates Breast Cancer Cell Migration through Trio and Rac. Genes Cancer. 2011;2(9):880-8.

36. Satriyo PB, Bamodu OA, Chen JH, Aryandono T, Haryana SM, Yeh CT, et al. Cadherin 11 Inhibition Downregulates beta-catenin, Deactivates the Canonical WNT Signalling Pathway and Suppresses the Cancer Stem Cell-Like Phenotype of Triple Negative Breast Cancer. J Clin Med. 2019;8(2):148.

37. Chen JH, Huang WC, Bamodu OA, Chang PM, Chao TY, Huang TH. Monospecific antibody targeting of CDH11 inhibits epithelial-tomesenchymal transition and represses cancer stem cell-like phenotype by up-regulating miR-335 in metastatic breast cancer, in vitro and in vivo. BMC Cancer. 2019;19(1):634.

38. Yuan S, Li L, Xiang S, Jia H, Luo T. Cadherin-11 is inactivated due to promoter methylation and functions in colorectal cancer as a tumour suppressor. Cancer Manag Res. 2019;11:2517-29.

39. Zhu Q, Wang Z, Zhou L, Ren Y, Gong Y, Qin W, et al. The role of cadherin-11 in microcystin-LR-induced migration and invasion in colorectal carcinoma cells. Oncol Lett. 2018;15(2):1417-22.

40. Lee YC, Cheng CJ, Huang M, Bilen MA, Ye X, Navone NM, et al. Androgen depletion up-regulates cadherin-11 expression in prostate cancer. J Pathol. 2010;221(1):68-76.

41. Tu SM, Lin SH. Clinical aspects of bone metastases in prostate cancer. Cancer Treat Res. 2004;118:23-46.

42. Chu K, Cheng CJ, Ye X, Lee YC, Zurita AJ, Chen DT, et al. Cadherin-11 promotes the metastasis of prostate cancer cells to bone. Mol Cancer Res. 2008;6(8):1259-67.

43. Satcher RL, Pan T, Bilen MA, Li X, Lee YC, Ortiz A, et al. Cadherin-11 endocytosis through binding to clathrin promotes cadherin-11-mediated migration in prostate cancer cells. J Cell Sci. 2015;128(24):4629-41.

44. Huang CF, Lira C, Chu K, Bilen MA, Lee YC, Ye X, et al. Cadherin-11 increases migration and invasion of prostate cancer cells and enhances their interaction with osteoblasts. Cancer Res. 2010;70(11):4580-9.

45. Lira CB, Chu K, Lee YC, Hu MC, Lin SH. Expression of the extracellular domain of OB-cadherin as an $\mathrm{Fc}_{\mathrm{C}}$ fusion protein using bicistronic retroviral expression vector. Protein Expr Purif. 2008;61(2):220-6.

46. Lee YC, Bilen MA, Yu G, Lin SC, Huang CF, Ortiz A, et al. Inhibition of cell adhesion by a cadherin-11 antibody thwarts bone metastasis. Mol Cancer Res. 2013;11(11):1401-11.

47. Satcher RL, Pan T, Cheng CJ, Lee YC, Lin SC, Yu G, et al. Cadherin-11 in renal cell carcinoma bone metastasis. PLoS One. 2014;9(2):e89880.

48. Carmona FJ, Villanueva A, Vidal A, Munoz C, Puertas S, Penin RM, et al. Epigenetic disruption of cadherin-11 in human cancer metastasis. J Pathol. 2012;228(2):230-40.

49. Kaur H, Phillips-Mason PJ, Burden-Gulley SM, Kerstetter-Fogle AE, Basilion JP, Sloan AE, et al. Cadherin-11, a marker of the mesenchymal phenotype, regulates glioblastoma cell migration and survival in vivo. Mol Cancer Res. 2012;10(3):293-304

50. Schulte JD, Srikanth M, Das S, Zhang J, Lathia JD, Yin L, et al. Cadherin-11 regulates motility in normal cortical neural precursors and glioblastoma. PLoS One. 2013;8(8):e70962.
51. Birtolo C, Pham H, Morvaridi S, Chheda C, Go VL, Ptasznik A, et al. Cadherin-11 Is a Cell Surface Marker Up-Regulated in Activated Pancreatic Stellate Cells and Is Involved in Pancreatic Cancer Cell Migration. Am J Pathol. 2017;187(1):146-55.

52. Jin G, Hong W, Guo Y, Bai Y, Chen B. Molecular Mechanism of Pancreatic Stellate Cells Activation in Chronic Pancreatitis and Pancreatic Cancer. J Cancer. 2020;11(6):1505-15.

53. Lin YL, Gui SL, Ma JG. Aberrant methylation of CDH11 predicts a poor outcome for patients with bladder cancer. Oncol Lett. 2015;10(2):647-52.

54. Chen MK, Chen ZJ, Xiao KH, Qin ZK, Ye YL, Wen WJ, et al. Predictive value of cadherin-11 for subsequent recurrence and progression in non-muscle invasive bladder cancer. Jpn J Clin Oncol. 2020;50(4):456-64.

55. Zhang JX, He WL, Feng ZH, Chen DL, Gao Y, He Y, et al. A positive feedback loop consisting of C12orf59/NF-kB/CDH11 promotes gastric cancer invasion and metastasis. J Exp Clin Cancer Res. 2019;38(1):164.

56. Eyvazi S, Khamaneh AM, Tarhriz V, Bandehpour M, Hejazi MS, Sadat ATE, et al. CpG Islands Methylation Analysis of CDH11, EphA5, and HS3ST2 Genes in Gastric Adenocarcinoma Patients. J Gastrointest Cancer. 2020;51(2):579-83.

57. Lechuga S, Amin PH, Wolen AR, Ivanov AI. Adducins inhibit lung cancer cell migration through mechanisms involving regulation of cell-matrix adhesion and cadherin-11 expression. Biochim Biophys Acta Mol Cell Res. 2019;1866(3):395-408.

58. Piao S, Inglehart RC, Scanlon CS, Russo N, Banerjee R, D'Silva NJ. CDH11 inhibits proliferation and invasion in head and neck cancer. J Oral Pathol Med. 2017;46(2):89-97.

59. Lemma SA, Kuusisto M, Haapasaari KM, Sormunen R, Lehtinen T, Klaavuniemi T, et al. Integrin alpha 10, CD44, PTEN, cadherin-11 and lactoferrin expressions are potential biomarkers for selecting patients in need of central nervous system prophylaxis in diffuse large B-cell lymphoma. Carcinogenesis. 2017;38(8):812-20.

60. Otto CM, Lind BK, Kitzman DW, Gersh BJ, Siscovick DS. Association of aortic-valve sclerosis with cardiovascular mortality and morbidity in the elderly. N Engl J Med. 1999;341(3):142-7.

61. Sung DC, Bowen CJ, Vaidya KA, Zhou J, Chapurin N, Recknagel A, et al. Cadherin-11 Overexpression Induces Extracellular Matrix Remodeling and Calcification in Mature Aortic Valves. Arterioscler Thromb Vasc Biol. 2016;36(8):1627-37.

62. Hutcheson JD, Chen J, Sewell-Loftin MK, Ryzhova LM, Fisher CI, Su YR, et al. Cadherin-11 regulates cell-cell tension necessary for calcific nodule formation by valvular myofibroblasts. Arterioscler Thromb Vasc Biol. 2013;33(1):114-20.

63. Fisher CI, Chen J, Merryman WD. Calcific nodule morphogenesis by heart valve interstitial cells is strain dependent. Biomech Model Mechanobiol. 2013;12(1):5-17.

64. Zhou J, Bowen C, Lu G, Knapp Iii C, Recknagel A, Norris RA, et al. Cadherin-11 expression patterns in heart valves associate with key functions during embryonic cushion formation, valve maturation and calcification. Cells Tissues Organs. 2013;198(4):300-10.

65. Bowler MA, Bersi MR, Ryzhova LM, Jerrell RJ, Parekh A, Merryman WD. Cadherin-11 as a regulator of valve myofibroblast mechanobiology. Am J Physiol Heart Circ Physiol. 2018;315(6):H1614-H26.

66. Bowen CJ, Zhou J, Sung DC, Butcher JT. Cadherin-11 coordinates cellular migration and extracellular matrix remodeling during aortic valve maturation. Dev Biol. 2015;407(1):145-57.

67. Clark CR, Bowler MA, Snider JC, Merryman WD. Targeting Cadherin-11 Prevents Notch1-Mediated Calcific Aortic Valve Disease. Circulation. 2017;135(24):2448-50

68. Abraham DJ, Varga J. Scleroderma: from cell and molecular mechanisms to disease models. Trends Immunol. 2005;26(11):587-95.

69. Schneider DJ, Wu M, Le TT, Cho SH, Brenner MB, Blackburn MR, et al. Cadherin-11 contributes to pulmonary fibrosis: potential role in TGF- $\beta$ production and epithelial to mesenchymal transition. FASEB J. 2012;26(2):503-12

70. Wu M, Pedroza M, Lafyatis R, George AT, Mayes MD, Assassi S, et al. Identification of cadherin 11 as a mediator of dermal fibrosis and possible role in systemic sclerosis. Arthritis Rheumatol. 2014;66(4):1010-21.

71. Pedroza M, Welschhans RL, Agarwal SK. Targeting of cadherin-11 decreases skin fibrosis in the tight skin-1 mouse model. PLoS One. 2017;12(11):e0187109.

72. Choi JS, Kim JK, Yang YJ, Kim Y, Kim P, Park SG, et al. Identification of cromolyn sodium as an anti-fibrotic agent targeting both hepatocytes and hepatic stellate cells. Pharmacol Res. 2015;102:176-83.

73. Weiskirchen R, Tacke F. Liver Fibrosis: From Pathogenesis to Novel Therapies. Dig Dis. 2016;34(4):410-22.

74. Ruan W, Pan R, Shen X, Nie Y, Wu Y. CDH11 promotes liver fibrosis via activation of hepatic stellate cells. Biochem Biophys Res Commun. 2019;508(2):543-9. 
75. Pedroza M, To S, Smith J, Agarwal SK. Cadherin-11 contributes to liver fibrosis induced by carbon tetrachloride. Plos One. 2019;14(7):e0218971

76. Lodyga M, Cambridge E, Karvonen HM, Pakshir P, Wu B, Boo S, et al. Cadherin-11-mediated adhesion of macrophages to myofibroblasts establishes a profibrotic niche of active TGF- $\beta$. Sci Signal. 2019;12(564):eaao3469

77. Black M, Milewski D, Le T, Ren X, Xu Y, Kalinichenko VV, et al. FOXF1 Inhibits Pulmonary Fibrosis by Preventing CDH2-CDH11 Cadherin Switch in Myofibroblasts. Cell Rep. 2018;23(2):442-58.

78. Franzè E, Monteleone I, Laudisi F, Rizzo A, Dinallo V, Di Fusco D, et al. Cadherin-11 Is a Regulator of Intestinal Fibrosis. J Crohns Colitis. 2020;14(3):406-17.

79. Johnson AM, Olefsky JM. The origins and drivers of insulin resistance. Cell. 2013;152(4):673-84.

80. Chang SK, Kohlgruber AC, Mizoguchi F, Michelet X, Wolf BJ, Wei K, et al. Stromal cell cadherin-11 regulates adipose tissue inflammation and diabetes. J Clin Invest. 2017;127(9):3300-12.

81. Vasanthakumar A, Moro K, Xin A, Liao Y, Gloury R, Kawamoto S, et al. The transcriptional regulators IRF4, BATF and IL-33 orchestrate development and maintenance of adipose tissue-resident regulatory $\mathrm{T}$ cells. Nat Immunol. 2015;16(3):276-85.

82. Boudoulas KD, Hatzopoulos AK. Cardiac repair and regeneration: the Rubik's cube of cell therapy for heart disease. Dis Model Mech. 2009;2(7-8):344-58.

83. Schroer AK, Bersi MR, Clark CR, Zhang Q, Sanders LH, Hatzopoulos $\mathrm{AK}$, et al. Cadherin-11 blockade reduces inflammation-driven fibrotic remodeling and improves outcomes after myocardial infarction. JCI Insight. 2019;4(18):e131545.

84. Rüter B, Wijermans PW, Lübbert M. DNA methylation as a therapeutic target in hematologic disorders: recent results in older patients with myelodysplasia and acute myeloid leukemia. Int $\mathrm{J}$ Hematol. 2004;80(2):128-35. 